\title{
AN EXACT UPPER BOUND FOR THE GROUND-STATE ENERGY OF THE TRIANGULAR ANTIFERROMAGNETIC LATTICE BY MEANS OF A RENORMALIZATION PROCEDURE
}

\author{
H.P. van de BRAAK, W.J. CASPERS and M.W.M: WILLEMSE \\ Department of Applied Physics, Twente University of Technology, Enschede, The Netherlands
}

Received 27 April 1978

\begin{abstract}
A renormalization procedure gives a rigorous upper bound for the ground-state energy per spin for the triangular antiferromagnetic lattice with Heisenberg interaction.
\end{abstract}

Recently renormalization techniques have offered new possibilities to calculate approximate values for the ground-state energy of antiferromagnetic spin $\frac{1}{2}$ systems $[1,2]$ or to give a rigorous upper bound for this energy [3]. In this letter we give an upper bound per spin for the triangular lattice with an antiferromagnetic Heisenberg interaction between nearest neighbours. Anderson [4] and Fazekas and Anderson [5] have already given estimates of the ground-state energy of this lattice and have discussed in some detail the character of the lowest state.

Crucial in our method is the introduction of a hexagonal Kadanoff cell [6], as shown in fig. 1. Each lattice site may correspond with any of the seven points of a cell and around one given cell neighbouring cells may be arranged in two different ways. Consequently each scaling can be performed in 14 different ways. This relatively large freedom for the triangular

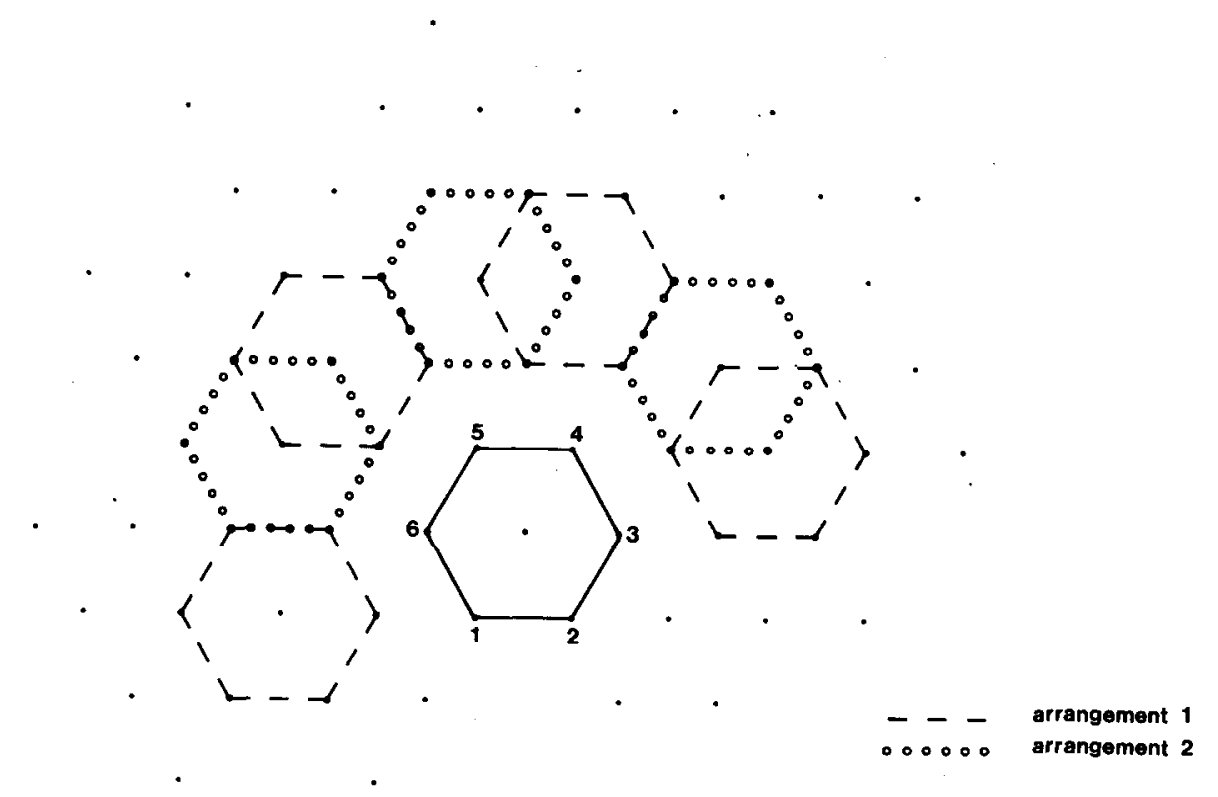

Fig. 1. Kadanoff cells in a triangular lattice. 
lattice is very suggestive of the idea that the quantummechanical ground state does not have a sublattice structure [4,5], but we do not intend to discuss this fundamental question any further in this letter.

The hamiltonian of the system

$$
H=J \sum_{\langle i, j\rangle} S_{i} \cdot S_{j}
$$

is a sum over nearest neighbour pairs $\langle i, j\rangle$ of lattice sites. An upper bound for the ground-state energy per spin is now determined by first calculating the ground. state energy per cell $[1,3]$. After that the secular part of the interaction between cells in their lowest state is evaluated. The ground state of a single cell is constructed from a stationary state of the corresponding hexagon (without central spin) because the energy of this ring, and its total spin, are constants of motion for the total cell. An elementary quantum-mechanical calculation results in a spin $S=1$ for the ring and a $\operatorname{spin} \bar{S}=\frac{1}{2}$ for a cell in the ground state. The energy is: $-\left(2+\frac{1}{2} \sqrt{5}\right) J$. The ground state is invariant for the discrete rotations about the hexagonal axis of the cell. The lattice of cells is isomorphic to the original lattice and their lowest states are Kramers doublets, so a renormalization of the hamiltonian $H$ can be performed immediately. One has to determine the matrix of the spin operators for the individual spins of a cell for the corresponding Kramers doublet.

This representation may be written in terms of the cell spins $S_{i}^{(1)}, i$ indicating a site in the lattice of cells. In this way we find that a spin of the ring (sites 1,2, $3, \ldots, 6$ in fig. 1) is equivalent to $\frac{2}{9} S_{i}^{(1)}$ for the lowest Kramers doublet. Taking into account that there exist three interacting spin pairs for two neighbouring cells we find an effective interaction between these cells in their lowest state of the form: $3(2 / 9)^{2} J S_{i}^{(1)} \cdot S_{j}^{(1)}$. So we immediately arrive at the following hamiltonian for the lattice of cells in their lowest Kramers states, $N$ denoting the total number of spins,

$H^{(1)}=-\frac{1}{7} N\left(2+\frac{1}{2} \sqrt{5}\right) J+\frac{4}{27} J \sum_{\langle i, j\rangle} S_{i}^{(1)} \cdot S_{j}^{(1)}$.

Because of $H^{(1)}$ being a projection of $H$ it gives an upper bound for the lowest eigenvalue of the latter. Repeating this procedure and taking into account that each step results in a lattice with a number of sites that equals $\frac{1}{7}$ of that of the previous one, we arrive at the following upper bound for the energy per spin:

$$
\begin{aligned}
-\frac{1}{7} & \left(2+\frac{1}{2} \sqrt{5}\right)\left[1+\frac{4}{27} \times \frac{1}{7}+\left(\frac{4}{27} \times \frac{1}{7}\right)^{2}+\ldots\right] J \\
\quad=-\frac{1}{7} & \frac{2+\frac{1}{2} \sqrt{5}}{1-4 / 189} J=-27 \frac{2+\frac{1}{2} \sqrt{5}}{185} J=-0.45506 J .
\end{aligned}
$$

This value should be compared with numerical results of the authors already cited [4,5]. First of all there is the expectation value for the pure Néel state with three different sublattices with magnetizations at an angle of $120^{\circ}$ to each other, which value equals the corresponding classical value and results in an energy per spin of $-0.375 J$ [4]. This value is also realized for the pair-bond state and is an upper bound. Rough calculations of the effects of zero-point fluctuations of spin waves gives: $(-0.463 \pm 0.007) J[4,7]$, whereas extrapolations of calculations with resonating singlet pair bonds result in eśtimates ranging from $(-0.54$ $\pm 0.01) \mathrm{J}$ (extrapolation from railroad trestle to the triangular lattice [4]) to $(-0.490 \pm 0.005) J$ (railroad trestle [4]), and $-0.46 J$ [5]. The last estimate of Fazekas and Anderson corresponds with the study of very large clusters with fluctuating pair bonds.

Our result gives an improvement of more than 20\% of the only rigorous upper bound that was known in the literature till now, i.e. the value $-0.375 \mathrm{~J}$, and does not differ much from the latest result of Fazekas and Anderson.

\section{References}

[1] H.P. van de Braak, W.J. Caspers, C. de Lange and M.W.M. Willemse, Physica 87A (1977) 354.

[2] R. Dekeyser, preprint.

[3] H.P. van de Braak, W.J. Caspers, F.W. Wiegel and M.W.M. Willemse, J. Stat. Phys., to be published.

[4] P.W. Anderson, Mat. Res. Bull. 8 (1973) 153.

[5] P. Fazekas and P.W. Anderson, Phil. Mag. 30 (1974) 423.

[6] L.P. Kadanoff, Physics 2 (1966) 263;

B. Widom, in: Fundamental problems in statistical mechanics, Vol. III, Proc. 1974 Wageningen Summer School, ed. E.G.D. Cohen'(North-Holland, Amsterdam, 1975) p. 1.

[7] F. Stern, Phys. Rev. 94 (1954) 1412;

R. Kubo, Rev. Mod. Phys. 25 (1953) 344. 